\title{
Time to failure of dynamic local load- sharing fiber bundle models in 1 to 3 dimensions
}

Miguel A. Clemente, Javier B. Gómez and Amalio F. Pacheco

Faculty of Sciences, University of Zaragoza, Pedro Cerbuna 12, 50009 Zaragoza, Spain

\section{Abstract}

Extensive Monte Carlo simulations are carried out in one, two and three dimensions for dynamic local load-sharing fiber bundle models following a power-law breaking rule with exponent $\rho$. This exponent controls de degree of disorder of the bundle. The results are obtained using two methods of introducing disorder in the simulations. In the standard, or classical, Monte Carlo method the disorder is quenched; in the second, or radioactive, method the disorder is annealed. Both methods give identical mean time-to-failure values for systems of the same size. However, the radioactive method proves to be more efficient due to the smaller standard deviation of the probability distribution function of the time-to-failure. We take advantage of this efficiency to compute the asymptotic mean time-to-failure of large systems as a function of the degree of disorder, as parameterized by $\rho$. Based on these extensive simulations, conclusions are drawn regarding the upper critical dimension of time-dependent local load-sharing fiber bundle models.

\section{Keywords}

Fiber-bundle models; local load-sharing; time-dependent failure; failure of disordered materials; Monte Carlo simulations; quenched disorder; annealed disorder; upper critical dimension

\section{Introduction}

Fiber-bundle models are simple tools for simulating the failure of heterogeneous materials from a microscopic viewpoint. They consist of a number of elements (called fibers) arranged in parallel and submitted to a load, either constant or time-dependent. The breaking properties of the elements are statistically distributed according to a specific probability distribution, and the aim is to assess the breaking properties of the whole ensemble (the bundle). The name of these models derives from its initial close connection with the strength of bundles of textile fibers [1]. Since Daniels' [1] seminal work, there has been a long tradition in the use of these models to 
analyze the microscopic details of the failure process of disordered materials (for a recent review, see Ref. [2]).

It was clear from the start that FBMs can be constructed to simulate either the static strength of materials or their failure by fatigue. The first type of FBMs aims at mimicking laboratory tests where samples (i.e., bundles) are subjected to an increasing load and the objective is to estimate the minimum load necessary to break the complete bundle. The second type of FBMs simulates breaking by delayed fatigue: individual fibers are assigned an initial time to failure which depends on the instantaneous load on each fiber and thus on the load history. The first type of model is known as static FBMs and the second type as dynamic or time-dependent FBMs. In this paper we deal with this second type of fiber-bundle model.

One of the key ingredients of FBMs, both static and dynamic, is the load transfer mechanism. As individual fibers break, the load that they were supporting is transferred to other intact fibers in the bundle. Depending on which fibers take this load, three basic load-transfer rules are defined: equal load-sharing, local load-sharing and hierarchical load-sharing.

Equal load-sharing (ELS) models are of a mean-field type and ignore the stress enhancements near locally failed regions. The name "equal load-sharing" stresses the fact that the load born by a failing element is transferred equally to all the remaining elements. Daniel's [1] seminal work on the strength of bundles of textile fibers was carried out precisely on static ELS bundles, work that was followed by Phoenix [3] and McCartney and Smith [4], who provided an improved recursive formula for the exact calculation of the probability of failure of a large bundle, and compare it with three different asymptotic approximations. Time-dependent ELS bundles were initially tackled by Bernard Coleman in a series of papers that dealt with specific cases [5-10], which were cast into a general theory of time-dependent ELS bundles in Refs. [3, $11]$.

The case where stress enhancements near the edge of the fracture are taken into account is known as the local load-sharing (LLS) rule, and this has proven much more difficult to address from an analytic point of view. In this type of load sharing only the nearest flanking survivors carry the extra load of a failed fiber. This was introduced in the literature by Scop \& Argon [12] and followed by Refs. [13-18] in the context of static bundles. This load transfer scheme is useful for modeling heterogeneous materials where friction between elements reduces the redistribution of stress to a local neighborhood, and has found a large field of application in manmade fiber-reinforced composites. The time-dependent version of these LLS models, to which this paper is dedicated, was first studied in Refs. [19-22], but advances here have been slow. Ref. [23] contains a review of the main findings together with new Monte Carlo simulations for a restricted version of the one-dimensional dynamic LLS model, whereas Ref. [24] provides 
analytic, asymptotic and Monte Carlo results for a generalized version of the same onedimensional model.

A third type of load sharing scheme,- hierarchical load-sharing (HLS), was introduced in the literature by Turcotte and collaborators $[25,26]$ in order to apply the fiber-bundle paradigm to large fractures (earthquakes). In the HLS scheme, the load of a failed element is redistributed via a hierarchical structure (fractal or Cayley tree) to a neighborhood whose size is of the same order as the size of the failed region. This seminal work was followed and expanded in Refs. $[27,28]$, who analyzed the properties of these static models by means of exact analytical techniques and by numerical simulations. Later, these results were extended to the timedependent HLS [29-32].

In summary, as there are two modalities of breaking (static and dynamic) and three basic schemes of load transfer (ELS, LLS, and HLS), this gives six different basic types of FBMs, each one with a different asymptotic behavior for large bundles: static ELS models have a critical point in the sense that there is a finite load $\sigma_{\mathrm{c}}$ below which an infinite bundle does not break and above which it breaks with a probability of one [1]; time-dependent ELS models also have a critical point, in this case in the form of a finite time of breaking for an infinite bundle [5]. On the other hand, neither the static nor the time-dependent one-dimensional LLS models have a critical point: both the strength of the static 1D LLS models [17, 33] and the time-tofailure of the time-dependent 1D models $[22,23]$ go to zero as the size of the bundle approaches infinity. Thus, we see that ELS and 1D-LLS models have a radically different asymptotic behavior, the former with finite strength and finite time to failure and the latter with zero strength and time to failure. In contrast, HLS models have a different behavior and, whereas static HLS models do not have a critical point (the strength tends to zero with increasing bundle size, albeit very slowly [28]), time-dependent ones do have a finite asymptotic time-to-failure, at least for systems under the power-law breaking rule and with small power-law exponents [29, $31,32]$.

Whereas in the ELS and HLS versions of the fiber-bundle models the spatial dimensionality does not matter in terms of the asymptotic behavior of the bundle, in the LLS case the dimensionality of the system plays a fundamental role and so we can define one-dimensional, two-dimensional, etc. versions of the LLS model. All the results summarized above pertain to the 1D version of the LLS models. This is so because little work has been done on higher dimensional time dependent LLS models regarding their asymptotic behavior (for static LLS models in higher dimensions, see, for example, Refs. [34, 35]). One exception in the case of time-dependent models is the work in Ref. [36], where the authors examined how the behavior of a bundle depends upon the range over which each fiber interacts with its neighbors, using a 
function that smoothly interpolates between pure ELS and pure LLS schemes. They performed 2D time-dependent simulations and found a crossover from mean-field (ELS) to short-range (LLS) behavior. In the region with LLS behavior they found a reduction of the time to failure with increasing system sizes, and they assumed that this behavior was identical to the 1D case, where the time to failure goes to zero as the size of the system increases to infinity. To the best of the authors' knowledge, no 3D time-dependent LLS simulations have been performed. Thus, an important question that we want to answer in this paper is: How does the asymptotic behavior of higher dimensional time-dependent LLS models compare to their 1D counterparts? From the computational point of view, two Monte Carlo methods have been devised to deal with dynamical FBMs. In the standard or classical Monte Carlo (cMC) method, a lifetime $t_{i 0}$ is assigned to each fiber at the beginning of the simulation drawn from a statistical distribution of lifetimes. Besides, each fiber bears a common load $\sigma_{0}$. When one of these elements fails, its load is transferred to the neighboring elements, increasing thus the load on them and reducing their initially assigned lifetimes. This process will be detailed in Section II. The point is that in the standard method the disorder is introduced at the beginning of the simulation, when the collection of lifetimes, $t_{i 0}$, is fixed. Afterwards, the process of gradual reduction of the bundle size until the failure of the last fiber is deterministic. Thus, in the standard Monte Carlo method the disorder is quenched.

In the other method, that we will call the radioactive Monte Carlo (rMC) method because of the parallelism between the process of radioactive decay and the fracture of a fiber bundle, the state of the system at any time is a vector containing the position of each surviving fiber and its individual load, $\sigma_{i}$. An individual breaking width $\Gamma_{i}$ is defined, which depends only on $\sigma_{i}$. Besides, a total width $\Gamma=\sum_{i} \Gamma_{i}$ of the surviving collection of fibers is calculated. The time taken by the next element to fail is $\delta=1 / \Gamma$, and the probability that the failed element be the $i$ th element is $p_{i}=\Gamma_{i} / \Gamma$. Thus, in this method it is necessary to compute a probability among the set of surviving elements, i.e., to define successive random numbers, at each step of breaking. Therefore, in this second method the disorder is annealed.

Our purpose in this paper is to perform extensive Monte Carlo simulations using the two Monte Carlo methods (cMC and rMC) with large time-dependent LLS bundles in order to compare their respective efficiency. As the simulations will be performed in one, two, and three spatial dimensions, from the results obtained we will be able to draw some conclusions about the upper critical dimension of the dynamic LLS models.

In Section II a brief description of the two methods of computation, the cMC and the rMC, is given. In Section III the efficiency of the two methods is compared. In Section IV the main 
results concerning the asymptotic behavior of large LLS bundles in 1D, 2D and 3D are presented and discussed in terms of the upper critical dimension. Finally, Section V contains the conclusions.

\section{The two computation methods}

\subsection{Standard Monte Carlo method}

As already mentioned in the Introduction, in the standard Monte Carlo approach (e.g., [29]) the population of initial fiber lifetimes is fixed at the beginning of the simulation and the subsequent breaking process is deterministic. In other words, the disorder is quenched: a random lifetime is assigned to each individual element at the onset, drawn from a probability distribution. This is the only time during the whole simulation where random numbers are utilized.

The failure of each element depends on its initial random lifetime and on its load history $\sigma(t)$. The probability $P(t ; \sigma)$ of a single fiber breaking at time $t$ after suffering the external load history $\sigma(t)$ is of the form $[3,9,23,29]$ :

$$
P(t ; \sigma)=1-\exp \left[-\Psi\left(\int_{0}^{t} \kappa[\sigma(\tau)] d \tau\right)\right], \quad t \geq 0
$$

In this equation the function $\Psi(x)$ is the hazard function and defines the shape of the probability distribution in terms of the integrated time. Inside the integral in Eq. (2) there is another quantity, $\kappa(\sigma)$, which is known as the breaking rule or hazard rate for the instantaneous failure of fibers. Two different breaking rules are commonly used in fiber-bundle models: the exponential breaking rule [5],

$$
\kappa(\sigma)=e^{\sigma / \sigma_{0}},
$$

and the power-law breaking rule [7],

$$
\kappa(\sigma)=\left(\sigma / \sigma_{0}\right)^{\rho}
$$

The exponential breaking rule is associated with time-dependent FBMs because it is the simplest form that gives first-order kinetics compatible with the weakest-link criterion [5]. The power-law breaking rule was introduced in the fiber-bundle paradigm to impart to Eq. (2) the commonly observed Weibull behavior of real materials under constant load [37], in conjunction with a hazard function $\Psi(x)$ of the form

$$
\Psi(x)=x^{\beta}, \quad x \geq 0
$$

where $\beta$ is a positive constant known as the Weibull index. Thus, if we substitute Eqs. (5) and 
(4) into Eq. (2), we have

$$
P(t ; \sigma)=1-\exp \left[-\left(\int_{0}^{t}\left[\sigma(\tau) / \sigma_{0}\right]^{\rho} d \tau\right)^{\beta}\right], \quad t \geq 0,
$$

and if we consider a constant load history $\sigma(\mathrm{t})=\sigma_{0}$, then

$$
P(t)=1-\exp \left(-t^{\beta}\right), \quad t \geq 0,
$$

which has the form of a Weibull function in the time variable. In what follows we will restrict ourselves to the case $\beta=1$, transforming the Weibull distribution into an exponential distribution. Taking $\beta=1$ renders a fiber memoryless [23], i.e., its remaining lifetime, given survival to time $t$, is independent of its load history up to time $t$, making the model simpler. In this paper we will assume that fibers follow a power-law breaking rule with exponent $\rho$, Eq. (4). This exponent is called the stress corrosion index in rock physics literature, and is typically in the range 2-5 for manmade structures [29] and 10-50 for rocks [38]. We will also take $\sigma_{0}=1$, making our load and time variables dimensionless.

Due to the load transfer from broken to unbroken elements, the individual load supported by the surviving elements grows during the progressive breakdown of the bundle, thus reducing their initial lifetimes and the time to failure of the whole bundle. This reduced time to failure of fiber $i, t_{i f}$, is given by

$$
t_{i 0}=\int_{0}^{t_{i f}} \sigma(t)^{\rho} d t
$$

where $t_{i 0}$ is the initial (random) lifetime of the $i$-th fiber and $\sigma(t)$ is its load history. To write this equation we have already used the power-law breaking rule and $\beta=1$ in Eq. (6).

For solving the problem by the classical Monte Carlo method, each element $i=1, \ldots, N$ in a system of size $N$ is initially assigned a random time to failure $t_{i 0}$ under load $\sigma_{0}=1$ based on Eq. (7). The actual time to failure $t_{i f}$ of element $i$ will be reduced below $t_{i 0}$ every time a load is transferred to this element due to the failure of other (neighboring) elements in the system. This reduced time to failure $t_{i f}$ is obtained by requiring that Eq. (8) is satisfied under the new load $\sigma(t)$. The $t_{i f}$ corresponding to the last failing element, the $N$-th, is the lifetime of the bundle, $T_{f}$. It is clear that the dynamics of the simulation is deterministic once the $N$ initial individual failure times have been assigned. Each realization of a random $N$-tuple of failure times will give a different time to failure $T_{f}$ for the bundle, and the mean value of this time to failure is obtained by averaging a sufficiently large number of simulations. 


\subsection{Radioactive Monte Carlo method}

This method of solving time-dependent fiber-bundle models was introduced in Refs. [31,39] in connection with the computer simulation of large HLS bundles, although the algorithm is general enough to be applied to any load-sharing scheme. In this method, as briefly summarized in the Introduction, the disorder is annealed as it is necessary to compute a probability of failure among the set of surviving elements in each step of the breaking process, and for that purpose a new random number is needed.

Initially, at $t=0$, the system is intact and all the $N$ elements support a load $\sigma(t=0)=\sigma_{0}=1$. The breakdown of the bundle proceeds sequentially, element after element, with the appropriate load redistribution after each breaking event, from $i=1$ until the breaking of the last, $i=N$, element. Thus, the $i$ index denotes the successive $N$ steps of the breaking process of the bundle. The point then is to determine how long it takes to break one element in the $i$ th step. This will be denoted by $\delta_{i}$ and its computation will be specified later. In consequence, the time of collapse of a bundle of $N$ fibers is

$$
T_{f}=\sum_{i=1}^{N} \delta_{i}
$$

In an arbitrary intermediate state $i$ of breaking, the bundle will be in a configuration with $i$ broken elements, and $N-i$ unbroken elements bearing different individual loads $\sigma_{j}$. The $j$ index extends to all $N-i$ unbroken elements of the bundle. Note that due to the conservation of the total load during the breaking and redistribution processes, at any $i, N \sigma_{0}=N=\sum_{j=1}^{N-i} \sigma_{j}$.

Now, in analogy with a radioactive process (where the concept of decay width is essential to compute the decay rate of an isotope with more than one decay mode), we define the breaking width of an unbroken element as

$$
\Gamma_{j}=\sigma_{j}^{\rho} .
$$

Note that $\Gamma_{j}$ is the same thing as $\kappa$ in Eq. (4). The total breaking width of the configuration at step $i$ can then be written as

$$
\Gamma(i)=\sum_{j=1}^{N-i} \Gamma_{j}
$$

The lifetime of that configuration is simply the inverse of the total breaking width (in complete analogy with the lifetime of a radioactive isotope, which lifetime is the inverse of the total decay width): 


$$
\delta_{i}=\frac{1}{\Gamma(i)}
$$

Finally, the probability of breaking the unbroken element $j$ is given by

$$
p_{j}(i)=\frac{\Gamma_{j}}{\Gamma(i)}
$$

which is, using again the terminology of radioactive decay, the branching ratio of the $j$ th path. Thus, the choice of the specific fiber that breaks in each time step is made by generating a random number between 0 and 1 . We see that using this approach we will obtain, as for the cMC, different values for the $N$ deltas in each Monte Carlo simulation of the total breaking of a bundle. The mean value of a sufficiently large number of simulations provides, again, a convergent result for $T_{f}$, the lifetime of the system. But in contrast to the $\mathrm{cMC}$, here one starts with a unique configuration, and the fluctuations in $T_{f}$ arise from the different rupture paths resulting in each simulation.

\subsection{The 1D-LLS algorithm and its generalization to higher dimensions}

The 1D-LLS model we have implemented follows the procedure of, e.g., Newman and Phoenix (2001), in which the 1D fiber bundle is a linear chain of $N$ fiber elements numbered from 1 to $N$. Each element is initially loaded with a load $\sigma_{0}$ (that is taken as unity) and the system evolves as explained in the previous two sections depending on which version of the Monte Carlo method is used. Once fiber failures have occurred, two different types of surviving fiber elements can be distinguished: interior surviving fibers and boundary surviving fibers. Interior surviving fibers are surrounded by surviving elements and thus carry the same initial load $\sigma_{0}$; on the other hand, boundary surviving fibers are those that are nearest neighbors to one or two failed fibers, and thus carry the extra load inherited from them. In general, a boundary surviving fiber that has $r$ failed fibers to its left and right carry a load

$$
\sigma_{r}=1+\frac{r}{2}
$$

This expression is valid for all fibers except those at both ends of the chain. Rule (14) has the property of giving the same extra load to all the boundary fibers of a crack (a crack is simply a contiguous segment of failed fibers), thus enabling the computation of the load of each boundary fiber without the need of specifying the complete load history of each fiber. In other words, given the geometrical configuration of the system at a given instant, the load of every surviving fiber can be computed without invoking the load history. This symmetry is compatible with fracture mechanics and the concept of the crack intensity factor and, at the same time, simplifies the internal bookkeeping of the Monte Carlo algorithm. 
This procedure can be generalized to two and more dimensions simply by redefining a crack as a cluster of contiguous failed nearest-neighbor fibers. In a 2D square lattice each fiber has four nearest neighbors while in a 3D cubic lattice it has six. Thus, the whole procedure can be boiled down to tracking the clusters of failed fibers (that is, the crack population) and assigning to all surviving boundary elements the extra load coming from its flanking cracks (clusters) of total size $n$ and perimeter $\ell, \sigma_{n, \ell}=1+n / \ell$, where the perimeter is the number of elements forming the boundary of the cracks. In a 2D square lattice the maximum number of flanking cracks is four, and in a 3D cubic lattice, six. For example, in a 2D square lattice a single broken element (crack o size $n=1$ ) has a perimeter $\ell=4$ and, thus, $\sigma_{n, \ell} \equiv \sigma_{1,4}=1+1 / 4$, as expected. Obviously, the necessity of tracking the evolution of all the clusters of failed fibers imposes a penalty on the computation needs of these higher dimensional LLS models in comparison with the simple, chain-like 1D-LLS bundles. However, the Hoshen-Kopelman cluster counting algorithm [40] is quite efficient and we have been able to simulate 2D and 3D systems with up to a million fibers with both Monte Carlo methods (cMC and rMC), although computing times are quite long, as the next section will show.

\section{Comparison of the two Monte Carlo methods}

Because the $\mathrm{cMC}$ and $\mathrm{rMC}$ are two different ways of solving a fiber bundle model by means of Monte Carlo simulations (where the key difference, although not the only one, pertains to the type of disorder, quenched disorder in the cMC and annealed disorder in the rMC), first we should check whether both Monte Carlo procedures deal with the same problem. As our main purpose here is to know how the mean time to failure, $\bar{T}_{f}$, changes with the system size $N^{D}$ (where $D$ is the dimensionality of the system), the fundamental variable for us is $\bar{T}_{f}$. We would say that both implementations of the Monte Carlo algorithm solve the same problem if they give the same value for $\bar{T}_{f}$.

Before centering the analysis in the mean value of $\bar{T}_{f}$ for different $\rho$ values and system sizes, we show in Figure 1, as an example, the internal breaking dynamics of 2D 30x30 systems with $\rho=5$. The graphs plot the time interval between successive breakings (the deltas), starting with the first element to break and ending with the last. The continuous line in each graph is the rMC result and the open circles the $\mathrm{cMC}$ result. The graph on the left is for one Monte Carlo realization, whereas the graph on the right is the average of 100 Monte Carlo realizations. Three conclusions are immediately apparent: (1) the rMC method has much less dispersion than the cMC method; (2) both methods give the same deltas; and (3) both methods give the same final time to failure. This last point is the most important for us and is quantified below for different 
spatial dimensions and different $\rho$ values.
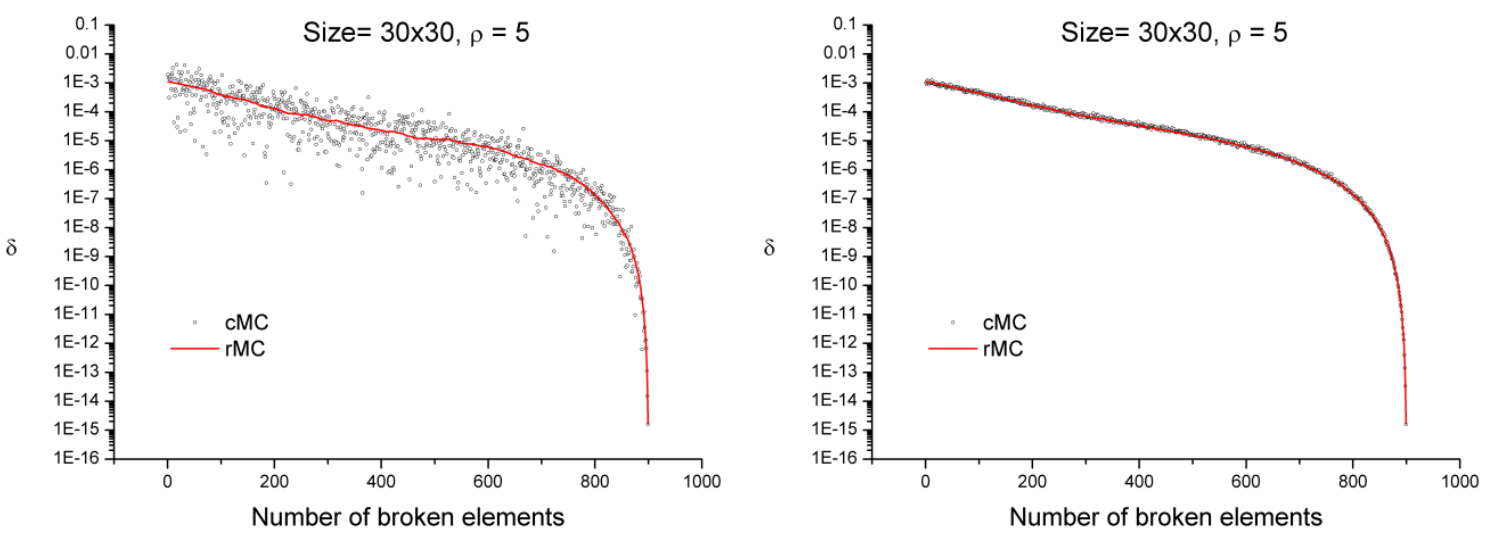

Figure 1. Internal breaking dynamics of 30x30 time-dependent 2D LLS systems for $\rho=5$. In the graphs the time interval between successive failures is plotted against the number of broken elements. The continuous line is the result of the rMC method and the open circles the result of the cMC method. The graph on the left is for one Monte Carlo realization, whereas the graph on the right averages 100 Monte Carlo realizations.

Table I summarizes the main results obtained with the two Monte Carlo implementations for LLS bundles of different sizes in 1D, 2D and 3D for two different values of the exponent $\rho$ ( $\rho=$ 2 and $\rho=20)$. The table gives the mean time to failure $\left(\bar{T}_{f}\right)$ and its standard deviation $( \pm 1 \sigma)$, together with the ratio of mean times to failure, $r\left(\bar{T}_{f}\right)=\left(\bar{T}_{f}\right)_{\mathrm{cMC}} /\left(\bar{T}_{f}\right)_{\mathrm{rMC}}$, and the ratio of standard deviations, $r(\sigma)=\sigma_{\mathrm{cMC}} / \sigma_{\mathrm{rMC}}$. As can be seen, the ratio of mean times to failure is always close to 1.0 (maximum deviation of $9 \%$ for the 1D-LLS case with $\rho=20$ and $N=$ 10000), which indicates that both Monte Carlo methods give essentially the same result for the variable $\bar{T}_{f}$. As for the standard deviation, the picture is quite different. The standard deviation ratio $r(\sigma)$ is always larger than one and for specific combinations of $D, N$ and $\rho$, the ratio is very high, indicating that the rMC has a much smaller standard deviation than the cMC. This is explicitly shown in Figure 2 where the empirical distribution functions for 2D-LLS bundles of size $N=50^{2}$ are depicted for $\rho=2$ (left) and $\rho=4$ (right). From the results gathered in Table I it can be concluded that the standard deviation ratio $r(\sigma)$ is larger for small systems and tends to diminish with system size (which is logical as the standard deviation should be zero for an infinite system). Also, $r(\sigma)$ is larger for smaller values of $\rho$ (again a logical results as $\rho$ parameterizes the disorder in the fibers' time to failure, and a larger $\rho$ means a smaller disorder). Finally, $r(\sigma)$ is also larger for 1D systems than for 2D or 3D systems. The only exception to these rules is the 2D-LLS case for $\rho=2$. Most of the difference in standard deviation between the $\mathrm{cMC}$ and $\mathrm{rMC}$ implementations comes from the much larger dispersion of delta values during the breaking of the system, as Figure 1 clearly shows. The much reduced dispersion in 
delta values of the rMC produces much more stable time to failure values, which translates directly into lower standard deviations around the mean value (and also into lower standard errors).

Table I. Comparison of the mean time to failure and standard deviation for the two Monte Carlo methods.

\begin{tabular}{|c|c|c|c|c|c|c|c|c|c|c|c|c|c|}
\hline \multirow[t]{3}{*}{ Dim } & \multirow[t]{3}{*}{ Size } & \multicolumn{6}{|c|}{$\rho=2$} & \multicolumn{6}{|c|}{$\rho=20$} \\
\hline & & \multicolumn{2}{|c|}{$\mathrm{cMC}$} & \multicolumn{2}{|c|}{ rMC } & \multirow{2}{*}{$\begin{array}{c}\mathrm{r}\left(\bar{T}_{f}\right. \\
) \\
\end{array}$} & \multirow[t]{2}{*}{$\mathrm{r}(\sigma)$} & \multicolumn{2}{|c|}{$\mathrm{cMC}$} & \multicolumn{2}{|c|}{ rMC } & \multirow{2}{*}{$\begin{array}{c}\mathrm{r}\left(\bar{T}_{f}\right. \\
)\end{array}$} & \multirow[t]{2}{*}{$\mathrm{r}(\sigma)$} \\
\hline & & $\bar{T}_{f}$ & $\pm 1 \sigma$ & $\bar{T}_{f}$ & $\pm 1 \sigma$ & & & $\bar{T}_{f}$ & $\pm 1 \sigma$ & $\bar{T}_{f}$ & $\pm 1 \sigma$ & & \\
\hline 1D- & 100 & 0.3476 & 0.054 & 0.3486 & 0.029 & 1.00 & 1.9 & 0.0108 & 0.011 & 0.0101 & $8.9 \mathrm{e}-6$ & 1.07 & 1236 \\
\hline \multirow[t]{2}{*}{ LLS } & 1000 & 0.2801 & 0.025 & 0.2779 & 0.022 & 1.01 & 1.1 & 0.00121 & 0.0011 & 0.00114 & $2.6 e-5$ & 1.06 & 42.3 \\
\hline & 10000 & 0.2354 & 0.0024 & 0.2320 & 0.0024 & 1.01 & 1.0 & 0.00023 & 0.0001 & 0.00021 & $3.4 e-5$ & 1.09 & 2.9 \\
\hline 2D- & $10^{2}$ & 0.4872 & 0.0056 & 0.4871 & 0.0037 & 1.00 & 1.5 & 0.0134 & 0.011 & 0.0133 & 0.00053 & 1.01 & 20.7 \\
\hline \multirow[t]{2}{*}{ LLS } & $50^{2}$ & 0.4782 & 0.010 & 0.4781 & 0.0011 & 1.00 & 9.1 & 0.0021 & 0.0009 & 0.0021 & 0.00051 & 1.00 & 1.8 \\
\hline & $100^{2}$ & 0.4778 & 0.0059 & 0.4778 & 0.0006 & 1.00 & 9.8 & 0.0013 & 0.0005 & 0.0013 & 0.00036 & 1.00 & 1.4 \\
\hline 3D- & $5^{3}$ & 0.4990 & 0.05 & 0.4989 & 0.0015 & 1.00 & 33.3 & 0.01897 & 0.010 & 0.01894 & 0.0013 & 1.00 & 7.7 \\
\hline \multirow[t]{2}{*}{ LLS } & $10^{3}$ & 0.4945 & 0.018 & 0.4945 & 0.0009 & 1.00 & 20.0 & 0.00928 & 0.002 & 0.00929 & 0.0014 & 1.00 & 1.4 \\
\hline & $20^{3}$ & 0.4940 & 0.004 & 0.4939 & 0.0003 & 1.00 & 13.3 & 0.00567 & 0.001 & 0.00599 & 0.0009 & 0.95 & 1.1 \\
\hline
\end{tabular}
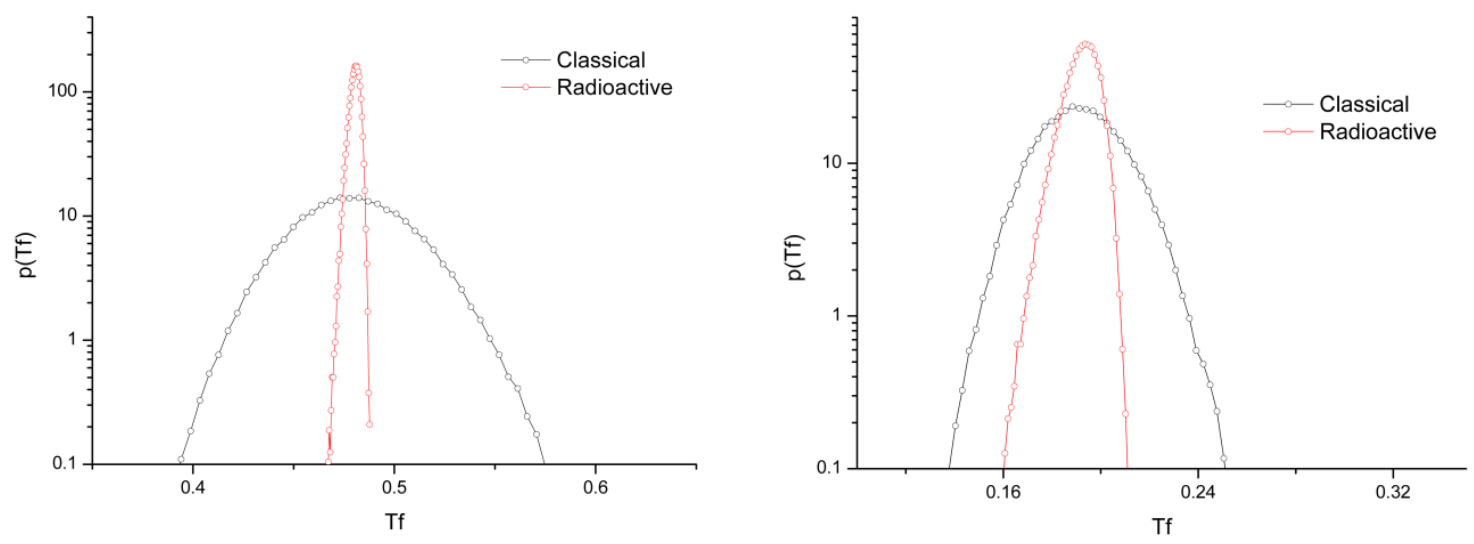

Figure 2. Comparison of the empirical distribution functions of the two Monte Carlo methods for two different values of the exponent $\rho$ in the 2D-LLS case.

Table II summarizes the efficiency differences between the $\mathrm{cMC}$ and $\mathrm{rMC}$ simulation methods as applied to time-dependent LLS bundles. We define the efficiency of a method as the number of simulations that must be performed, for a given $N$ and $\rho$, to achieve a relative error of $0.1 \%$ in the mean value of the time to failure, $\bar{T}_{f}$. From the standard error of the mean, SE, which gives the absolute error, we can compute the relative error by dividing the standard error by the mean value, $\mathrm{SE} / \bar{T}_{f}$. If we want this relative error to be $0.1 \%=0.001$, then we set $\mathrm{SE} / \bar{T}_{f}=0.001$, and recalling the definition of standard error, $\mathrm{SE}=\mathrm{SD} / \sqrt{n}$, where $\mathrm{SD}$ is the standard deviation and $n$ the number of simulations, we have $S D /\left(\bar{T}_{f} \sqrt{n_{0.1 \%}}\right)=0.001$ and thus:

$$
n_{0.1 \%}=\left(\frac{\mathrm{SD}}{0.001 \bar{T}_{f}}\right)^{2}
$$

This quantity is the one given in Table I, together with the time per simulation, tps, in seconds, 
for both Monte Carlo methods and two different values of the $\rho$ exponent, $\rho=2$ and $\rho=20$.

As the tps for $\mathrm{cMC}$ and $\mathrm{rMC}$ are very similar, the difference in efficiency between both MC methods stems mainly from the difference in standard deviation, as Figure 2 clearly shows.

Table II. Number of simulations to reach an error of $0.1 \%$ in the mean time to failure (n) and time per simulation (tps) in seconds. The efficiency ratio $\varepsilon$ is $\left(n \times \mathrm{tps}_{\mathrm{cMC}} /(n \times \mathrm{tps})_{\mathrm{rMC}}\right.$.

\begin{tabular}{|c|c|c|c|c|c|c|c|c|c|c|c|}
\hline \multirow[t]{3}{*}{ Dim } & \multirow[t]{3}{*}{ Size } & \multicolumn{5}{|c|}{$\rho=2$} & \multicolumn{5}{|c|}{$\rho=20$} \\
\hline & & \multicolumn{2}{|c|}{$\mathrm{cMC}$} & \multicolumn{2}{|c|}{$\mathrm{rMC}$} & & \multicolumn{2}{|c|}{$\overline{c M C}$} & \multicolumn{2}{|c|}{$\mathrm{rMC}$} & \multirow[b]{2}{*}{$\varepsilon$} \\
\hline & & $\mathrm{n}$ & tps & $\mathrm{n}$ & tps & $\varepsilon$ & $\mathrm{n}$ & tps & $\mathrm{n}$ & tps & \\
\hline 1D- & 100 & 24,367 & 0.0011 & 7063 & 0.0011 & 3.4 & 953,982 & 0.0027 & 0.60 & 0.0020 & $2.1 \mathrm{e} 6$ \\
\hline \multirow[t]{2}{*}{ dLLS } & 1000 & 7989 & 0.031 & 6324 & 0.028 & 1.4 & 818,131 & 0.17 & 370 & 0.104 & 3614 \\
\hline & 10000 & 10,767 & 2 & 10,550 & 2.15 & 0.9 & 185,694 & 14.1 & 40,800 & 8.77 & 7.3 \\
\hline 2D- & $10^{2}$ & 13,371 & 0.0012 & 56 & 0.0012 & 239 & 643,134 & 0.0026 & 1596 & 0.0026 & 403 \\
\hline \multirow[t]{2}{*}{ dLLS } & $50^{2}$ & 540 & 0.2 & 6.0 & 0.18 & 100 & 194,700 & 0.93 & 56,898 & 0.932 & 3.4 \\
\hline & $100^{2}$ & 153 & 3.1 & 1.6 & 2.96 & 100 & 140,990 & 14.8 & 79,149 & 15.13 & 1.7 \\
\hline 3D- & $5^{3}$ & 10,597 & 0.0018 & 14.5 & 0.0016 & 822 & 261,366 & 0.0034 & 4809 & 0.0026 & 71 \\
\hline \multirow[t]{2}{*}{ dLLS } & $10^{3}$ & 1357 & 0.057 & 3.1 & 0.037 & 674 & 72,317 & 0.15 & 22,454 & 0.102 & 4.7 \\
\hline & $20^{3}$ & 58 & 3.18 & 0.44 & 2.37 & 177 & 46,125 & 11 & 24,938 & 6.26 & 3.2 \\
\hline
\end{tabular}

\section{Discussion: Critical dimension, illusion or reality?}

It is clear that in load-transfer models, the larger the number of fibers that receive the load from a failing element (i.e., the load-transfer neighborhood), the more stable the system is. In LLS models the load-transfer neighborhood is related to the spatial dimension of the system, and in the HLS models to the coordination of the Cayley tree [29]. This means that the same LLS model in two spatial dimensions is more stable than in one spatial dimension and, correspondingly, the same models in three dimensions are more stable than in one or two dimensions, etc.

In 2D-LLS models, this was put in evidence in a series of papers by Herrmann and collaborators, both in static [41] and dynamic [36, 42] FBMs. It was assumed that the load transfer function had a variable spatial range parameterized as $\sigma_{\text {add }}=r^{-\gamma}$, where $\sigma_{\text {add }}$ is the load transferred to a neighbor element and $\gamma$ the exponent that controls the interaction range: as $\gamma \rightarrow 0$ the ELS case is recovered and as $\gamma \rightarrow \infty$ the LLS case is recovered. In this model the two regimes are separated by $\gamma_{\mathrm{c}} \cong 2$. When $\gamma<\gamma_{\mathrm{c}}$ the system has a large load-transfer neighborhood and behaves as an ELS bundle, whereas for $\gamma>\gamma_{c}$ the system has a small load-transfer neighborhood and its behavior is that of an LLS bundle, with a rather abrupt transition between both regimes.

However, the number of fibers that share the load of a failing element is not the only crucial factor regarding the stability of these models. The load enhancement induced near the fractures together with the assumed power-law behavior of the breaking width, $\Gamma=\sigma^{\rho}$, lead to a fast collapse of the bundle, especially for large values of the exponent $\rho$. In other words, it is not the same to assume $\rho=2$ as to assume $\rho=10$. The increase in this parameter can give rise not 
only to quantitative changes in the behavior of the bundle but also to qualitative changes. The essential role played by a parameter like $\rho$ in models close in spirit to FBMs can be seen in, for example, in Refs. [27, 43, 44]. If instead of using the power-law breaking rule, Eq. (4), one uses the exponential breaking rule, Eq. (3), the risk of fast, almost instantaneous failure can be even more extreme.

Keeping in mind these general thoughts regarding the key role that a parameter like $\rho$ can have in the fracture of disordered materials, we will now describe the behavior of time-dependent LLS bundles as a function of $\rho$ (which parameterizes the degree of disorder) and the spatial dimension $D$.

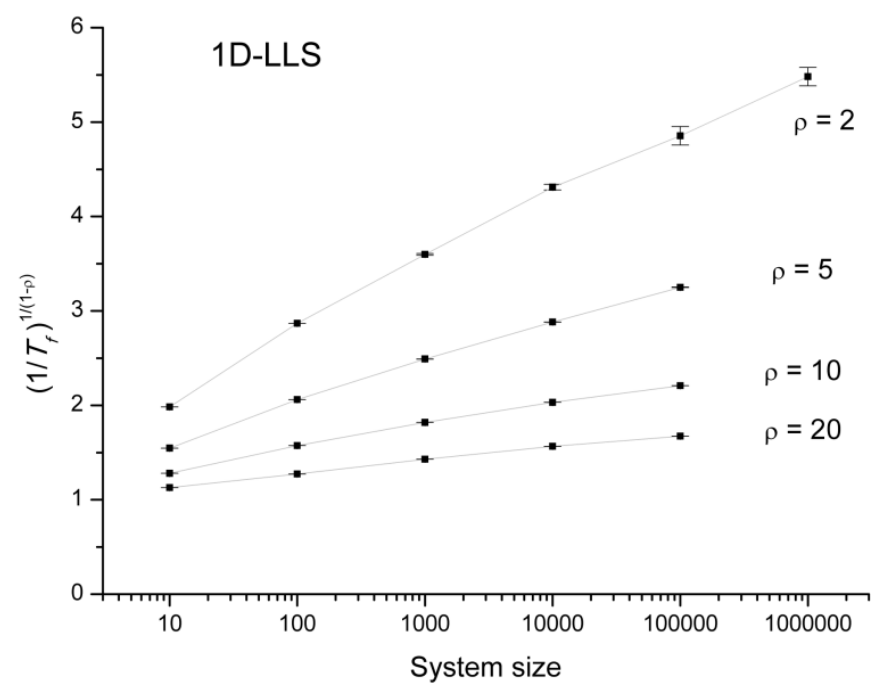

Figure 3. Asymptotic behavior of dynamic 1D-LLS systems for four different values of $\rho$. Simulations have been performed with the rMC method.

In Figure 3, $\left(1 / T_{f}\right)^{1 /(\rho-1)}$ is plotted against the system size (in log scale) for four different values of $\rho$ in 1D-LLS bundles. Although there is no exact formulation of the shape of the distribution function for the bundle lifetime, it is known that the mean time to failure of time-dependent 1DLLS models goes to zero as $1 / \ln N[22,23]$. In Ref. [23] the authors give approximate asymptotic results for the size effect on the mean bundle lifetime $\bar{T}_{f}$ for time dependent 1DLLS under the power-law breaking rule and $\beta=1$ (and for arbitrary $\beta$ in Ref. [24]):

$$
\bar{T}_{f} \propto 2^{\rho-1} \sigma_{0}^{-\rho}\left(\frac{\rho-1}{\ln N}\right)^{\rho-1} .
$$

From this equation it is immediately clear that a plot of $\left(1 / \bar{T}_{f}\right)^{1 /(\rho-1)}$ versus $\ln N$ is a straight line with a slope $1 / 2(\rho-1)^{-1}$. However, this slope is only reached for very large bundles and the actual slope for finite bundles has a correction $\left(1+\varepsilon_{N}\right)$, where $\varepsilon_{N}$ depends both on $N$ and $\rho$, tending to zero when $N \rightarrow \infty$ but increasing, for a given $N$, when $\rho$ increases. This correction 
introduces a curvature in the $\left(1 / \bar{T}_{f}\right)^{1 /(p-1)}$ versus $\ln N$ plot, as Figure 3 shows. The simulation results shown in the figure are consistent with the size-dependent slopes predicted by Eq. (16) with the $\left(1+\varepsilon_{N}\right)$ correction, confirming that the $\mathrm{rMC}$ method gives the same mean time to failure as the cMC method.

Figure 4 shows the simulation results for the 2D-LLS case. Although for $2 \mathrm{D}$ systems there is no analytical prediction of the asymptotic behavior of the time-dependent LLS model, we have used the same scaling for the plot axes as in Figure 3 in order to compare the asymptotic behavior of the 1D- and 2D-LLS bundles. What we see is a crossover from a behavior similar to the 1D-LLS systems for large values of $\rho(\rho>6)$, to an asymptotic behavior where $\left(1 / \bar{T}_{f}\right)^{1 /(\rho-1)}$ tends to reach a plateau. In other words, this crossover signals a change from a zero to a nonzero time to failure for infinite systems. This small- $\rho$ behavior is reminiscent of the behavior of dynamic ELS systems, whose time to failure for infinite systems is finite and equal to $1 / \rho$. For example, for $\rho=2$, the time to failure of an ELS bundles is 0.5 (and thus $\left(1 / \bar{T}_{f}\right)^{1 /(\rho-1)}=2$ ), whereas the asymptotic time to failure of a 2D-LLS bundle is $0.4778 \pm 0.0001,4.5 \%$ less ( $\left.\left(1 / \bar{T}_{f}\right)^{1 /(\rho-1)}=2.093 \pm 0.0001\right)$. In Figure 5 we have plotted only the case $\rho=2$ in order to see more clearly the plateau reached by the time to failure in systems with large disorder. The left graph plots the evolution with respect to variable $\left(1 / \bar{T}_{f}\right)^{1 /(\rho-1)}$ and the right graph in terms of the original variable $\bar{T}_{f}$. In both cases it is clear that the horizontality of the curve for $N>10,000$.

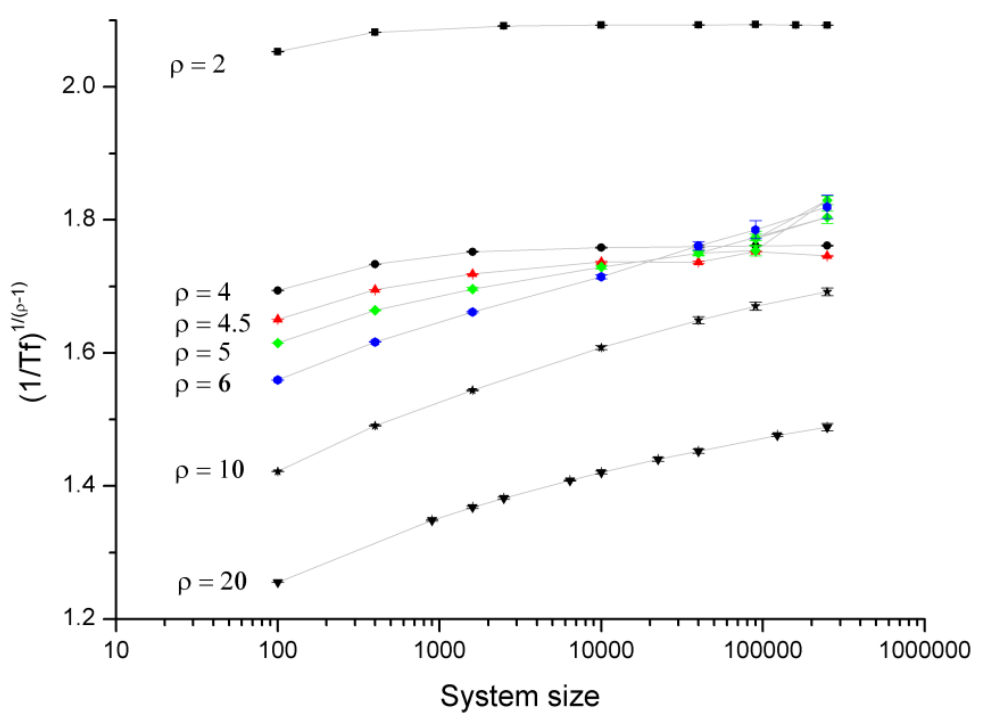

Figure 4. Asymptotic behavior of 2D-LLS bundles for increasing values of $\rho$, from $\rho=2$ to $\rho=20$. Note the apparent change in asymptotic behavior for $\rho$-values in the range 4-6. 

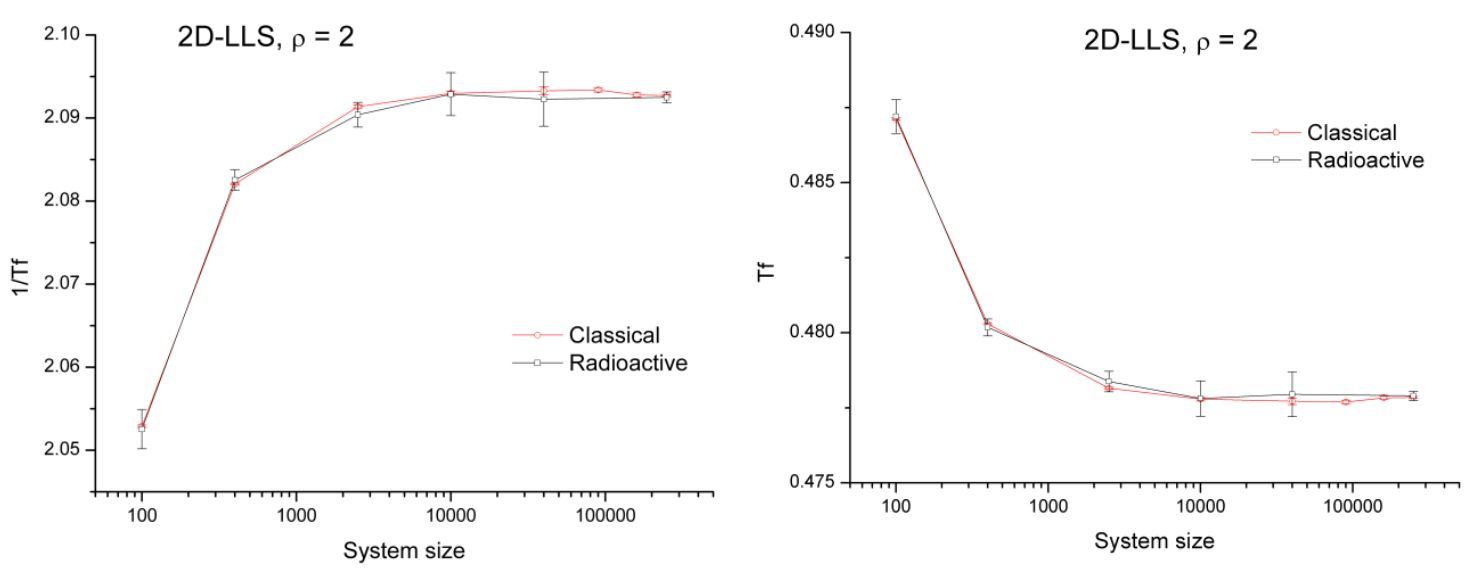

Figure 5. Asymptotic behavior of a 2D-LLS bundle with $\rho=2$. The left plot shows the trend in the $1 / T_{f}$ scale (in which 1D-LLS systems plot as straight lines with positive slope), and the right plot in the simple $T_{f}$ scale (in which the 1D-LLS systems tend to zero). It is obvious that the behavior shown for the $\rho=2$, 2D-LLS systems is radically different.

Finally, we have performed Monte Carlo simulations in 3D for three different values of $\rho: \rho=2$ and 4 as representative of high disorder materials and $\rho=20$ as representative of low disorder materials. Figure 6 plots the asymptotic behavior for the three $\rho$ values. As was the case for $2 \mathrm{D}$ LLS bundles, the $\rho=2$ and $\rho=4$ simulations tend to a constant mean time to failure as the system size is increased. For $N=20^{3}$ systems the time to failure for $\rho=2$ reaches a plateau at $T_{f}$ $=0.4939 \pm 0.0001$ or $\left(1 / \bar{T}_{f}\right)^{1 /(\rho-1)}=2.0247 \pm 0.0001$. This value of $T_{f}$ is only $1.2 \%$ smaller than the asymptotic time to failure for an ELS bundle with the same $\rho$. If we remember that 2D-LLS bundles have an asymptotic mean time to failure of $T_{f}=0.4778 \pm 0.0001$ for $\rho=2$, we see that the increase in spatial dimension brings the asymptotic time to failure closer to the mean field case, for which $T_{f}=1 / \rho=0.5$. In the case of $\rho=4$ we have a similar picture: the 3D simulations give an asymptotic time to failure of $T_{f}=0.22784 \pm 0.00002$ or $\left(1 / \bar{T}_{f}\right)^{1 /(\rho-1)}=1.63726 \pm 0.0005$, whereas the ELS value is $T_{f}=1 / \rho=0.25$., or $\left(1 / \bar{T}_{f}\right)^{1 /(\rho-1)}=1.5874$; i.e., the LLS value is $9.7 \%$ smaller than the ELS one. For comparison, the 2D asymptotic time to failure for $\rho=4$ is $T_{f}=$ $0.18304 \pm 0.0002$, a $36 \%$ smaller than the ELS value. These results gives weight to the idea that, at least for small- $\rho$ bundles, $D=2$ seems to be the critical dimension of time-dependent LLS bundles under the power-law breaking rule with an exponential hazard function (i.e., $\beta=1$ in Eqs. (5), (6) and (7)).

The $\rho=20$ case (Figure 6), in contrast, has an asymptotic behavior reminiscent of the one- and two-dimensional cases (see Figure 3 and Figure 4), with a monotonic increase in the inverse mean time to failure as the system size is increased, with no sign of a plateau. 


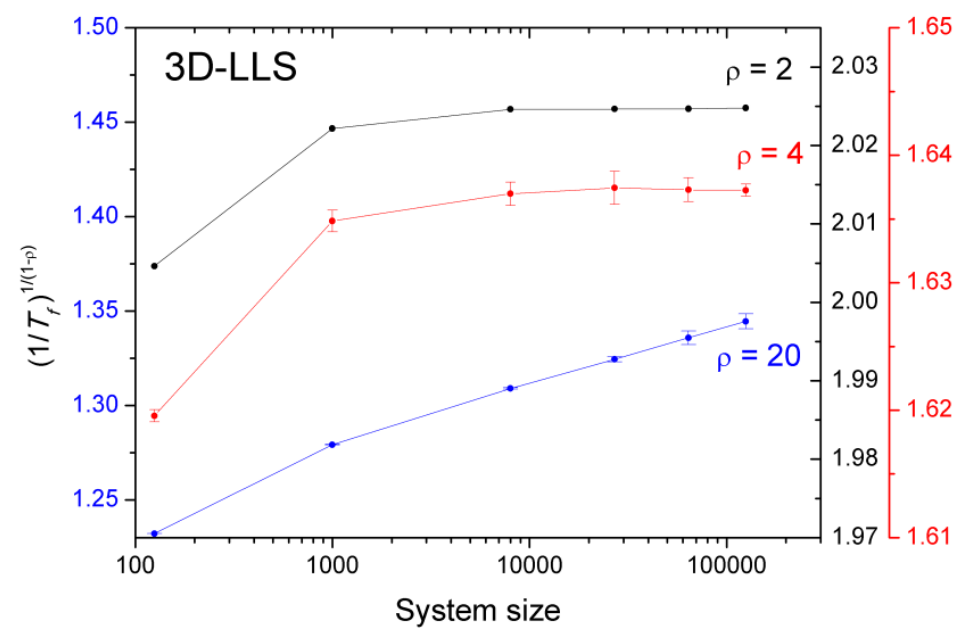

Figure 6. Asymptotic behavior of 3D-LLS bundles for three different values of $\rho$. The left axis corresponds to $\rho=20$ and the displaced right axis to $\rho=4$.

\section{Conclusions}

From the simulations performed in 1D-, 2D-, and 3D-LLS bundles, we draw a first conclusion: in the computation of time-dependent LLS models the radioactive implementation of the Monte Carlo method (rMC) is more efficient that the classical Monte Carlo method (cMC). The higher efficiency of the rMC is mainly due to the smaller standard deviation associated with the computation of the mean time to failure, $\bar{T}_{f}$.

Regarding the second question posed in the Introduction about the existence or not of a critical dimension in these models, our conclusion cannot be firm. Our results indicate that 2 seems to be a critical dimension for low values of $\rho$ (i.e., large levels of disorder), but not for high values of $\rho$ (i.e., small levels of disorder). This conclusion also holds for 3D simulations.

Retrospectively, this second conclusion leads us to rethink old beliefs. For example, in Ref. [32] we obtained a convincing numerical result indicating that time-dependent HLS bundles had a critical point, that is, a non-zero time to failure for infinite systems. In contrast, our results here regarding $\rho$ indicate that the existence of a critical point for $\rho=2$ does not guarantee the existence of a critical point for larger values of $\rho$.

After having explained the two Monte Carlo methods, it is clear that one could start a simulation using the cMC implementation as a first stage and, at a given point in the breaking process, pass on to the other implementation and finish the breaking of the bundle with the rMC. The information about the positions of the surviving elements and their loads as obtained with the $\mathrm{cMC}$, together with the time elapsed since the beginning of the process, would constitute the initial conditions for the second stage of this hybrid method. 


\section{References}

[1] Daniels HE, (1954). "The statistical theory of the strength of bundles of threads". Proc. Roy. Soc. A183, 404.

[2] Hansen A, Hemmer PC and Pradhan S (2015). The Fiber Bundle Model: Modeling Failure in Materials, Wiley-VCH Verlag, Weinheim, Germany.

[3] Phoenix SL (1978). Stochastic strength and fatigue of fiber bundles. International Journal of Fracture 14: $327-344$.

[4] McCartney LN and Smith RL (1983). Statistical theory of the strength of fiber bundles. J. Appl. Mech. 50: 601-608.

[5] Coleman BD (1957a). Time dependent of mechanical breakdown in bundles of fibers, I. Constant total load.

[6] Coleman BD (1957b). Time dependent of mechanical breakdown in bundles of fibers, II. The infinite ideal bundle under linearly increasing loads.

[7] Coleman BD (1958a). Time dependent of mechanical breakdown in bundles of fibers, III. The powerlaw breakdown rule.

[8] Coleman BD (1958b). Time dependent of mechanical breakdown in bundles of fibers, IV. Infinite ideal bundle under oscillating loads.

[9] Coleman BD (1958c). Statistics and time dependence of mechanical breakdown in fibers. [Here Coleman gives a general theory that includes all the special cases treated in the previous series of papers]

[10] Coleman BD (1959). Time dependent of mechanical breakdown in bundles of fibers, V. Fibers of Class A-2.

[11] Phoenix SL (1979). The asymptotic distribution of the time to failure of a fiber bundle. Adv. Appl. Prob. 11: 153-187.

[12] Scop PM, and Argon AS (1969). Statistical theory of strength of laminated composites. J. Composite Mat. 3: 30-47.

[13] Harlow DG, SL Phoenix (1978a). The chain-of-bundles probability model for the strength of fibrous materials I: analysis and conjectures. Journal of composite materials 12: 195-214.

[14] Harlow DG, SL Phoenix (1978b). The chain-of-bundles probability model for the strength of fibrous materials II: a numerical study of convergence. Journal of composite materials 12: 314-334.

[15] Harlow DG, SL Phoenix (1981a). Probability distributions for the strength of composite materials I: two-level bounds. International Journal of Fracture 17: 347-372.

[16] Harlow DG, SL Phoenix (1981b). Probability distributions for the strength of composite materials II: a convergent sequence of tight bounds. International Journal of Fracture 17: 601-630.

[17] Smith RL (1980). A Probability Model for Fibrous Composites with Local Load Sharing. Proc. R. Soc. London, Ser. A 372: 539-553.

[18] Phoenix SL, and Smith RL (1983). A comparison of probabilistic techniques for the strength of fibrous materials under local load-sharing among fibers. Int. J. Solid Struct. 19: 479-496.

[19] Tierney L (1982). Asymptotic bounds on the time to fatigue failure of bundles of fibers under local load sharing. Adv. Appl. Prob. 14: 95-121.

[20] Tierney L (1984), A probability model for the time to fatigue failure of a fibrous composite with local load sharing, Stochastic Processes and ther. Application 18: 139-152.

[21] Phoenix SL and Tierney L (1983), A statistical model for the time dependent failure of unidirectional composite materials under local elastic load-sharing among fibers, Engineering Fracture Mechanics 18: 193-215.

[22] Kuo CC and S.L. Phoenix (1987), Recursions and limit theorems for the strength and lifetime distributions of a fibrous composite. J. Appl. Prob.24: 137

[23] Newman WI and Phoenix SL (2001). Time-dependent fiber bundles with local load sharing. Phys. 
Rev. E63: 021507.

[24] Phoenix SL and Newman WI (2009). Time-dependent fiber bundles with local load sharing. II. General Weibull fibers. Phys. Rev. E 80: 066115.

[25] Turcotte DL, Smalley RF, and Solla SA (1985). Collapse of loaded fractal trees. Nature, 313, 671672.

[26] Smalley RF, Turcotte DL and Solla SA (1985). A renormalization group approach to the stick-slip behavior of faults. J. Geophys. Res., 90, 1894-1900.

[27] Sornette (1989), Failure thresholds in hierarchical and Euclidian space by real space renormalization group. Journal de Physique 50 (7): 745-755.

[28] Newman WI and Gabrielov AM (1991), Failure of hierarchical distributions of fibre bundles. I, Int. J. Fract. 50, 1-14.

[29] Newman I, Gabrielov AM, Durand TA, Phoenix SL, and Turcotte DL (1994). An exact renormalization model for earthquakes and material failure: statics and dynamics. Physica D 77: 200216.

[30] Newman WI, Turcotte DL and Gabrielov AM (1995), Log-periodic behavior of a hierarchical failure model with applications to precursory seismic activation, Phys. Rev. E 52, 4827.

[31] Vázquez-Prada M, Gómez JB, Moreno Y and Pacheco AF (1999). Time to failure of hierarchical load-transfer models of fracture. Phys. Rev. E 60: 2581-2594.

[32] Gómez JB and Pacheco AF (2006). Monte Carlo results in time-dependent hierarchical fiber-bundle models of fracture. Phys. Rev E 73: 047104.

[33] Gómez JB, Íñiguez D and Pacheco AF (1993). Solvable fracture model with local load transfer. Phys. Rev. Lett. 71: 380-383.

[34] Mahesh S, Phoenix SL and Beyerlein IJ (2002). Strength distributions and size effects for 2D and 3D composites withWeibull Pbers in an elastic matrix. International Journal of Fracture 115: 41-85.

[35] Sinha S, Kjellstadli JT, and Hansen A (2015). Local load-sharing fiber bundle model in higher dimension. Phys. Rev. E 92: 020401(R).

[36] Yewande OE, Moreno Y, Kun F, Hidalgo RC and Herrmann HJ (2003). Time evolution of damage under variable ranges of load transfer. Phys. Rev. E 68, 026116.

[37] Weibull W (1939). A statistical theory of the strength of materials. Proc. Ing. Vetenskapsakad. 151.

[38] Atkinson BK and Meredith PG (1987). The theory of subcritical crack growth with applications to minerals and rocks, In: BK Atkinson (ed), Fracture Mechanics of Rocks, Academic Press: 111-166.

[39] Gómez JB, Vázquez-Prada M, Moreno, Y and Pacheco AF (1999). Bounds to the time to failure of hierarchical systems of fracture, Phys. Rev. E 59: R1287-R1290.

[40] Hoshen, J. and Kopelman, R. (1976). Percolation and cluster distribution. I. Cluster multiple labeling technique and critical concentration algorithm. Physical Review B 14(8): 3438.

[41] Hidalgo RC, Moreno Y, Kun F and Herrmann HJ (2002). Fracture model with variable range of interaction. Phys. Rev. E 65, 046148.

[42] Hidalgo RC, Kun F and Herrmann HJ (2005). Slow relaxation of fiber composites, variable range of interaction approach. Physica A 347: 402-410.

[43] Andersen JV, Sornette D and Leung Kt (1997). Tricritical Behavior in Rupture Induced by Disorder. Phys. Rev. Lett. 78: 2140-2143.

[44] Moreno Y, Gómez JB and Pacheco AF (1999). Self-organized criticality in a fibre-bundle-type model. Physica A 274: 400-409. 